\title{
Sicherheitspolitik versus Menschenrechtsschutz?
}

\author{
Barbara Lochbihler*
}

\begin{abstract}
More than ever, in the aftermath of September $11^{\text {th }} 2001$, states are using the »war on terror « as a justification to dilute internationally recognised human rights standards. Increasing numbers of constitutional democratic governments argue that in the face of terrorist threats, citizens must sacrifice their rights so that the state can guarantee their security. Such a deterioration of human rights standards cannot be justified under any circumstances and in fact, the security of all people can only be achieved if universal human rights are guaranteed for all. Therefore, it must be the aim of every state to extend human rights protection, rather than restricting rights in the name of security.
\end{abstract}

Keywords: Menschenrechtsschutz, Sicherheit, Terrorismus

$\mathrm{O}$ bwohl die Relativierung schwerer Menschenrechtsverletzungen im Namen vermeintlich notwendiger Sicherheitsinteressen nicht neu ist und seit Jahrzehnten weltweit Angehörige von Minderheiten und politische Oppositionelle im Namen der nationalen Sicherheit bestraft, gefoltert und ihrer elementarsten Grundrechte beraubt wurden und werden, sieht amnesty international insbesondere seit dem 11. September 2001 die Gefahr einer Verschlechterung der bisher erreichten Menschenrechtsstandards auf der Basis der Allgemeinen Erklärung der Menschenrechte. Offener denn je missbrauchen Staaten den »Krieg gegen den Terror « als Rechtfertigung, um Menschen »verschwinden « $\mathrm{zu}$ lassen, um sie ohne ordentliches Verfahren zu inhaftieren, um sie zu misshandeln und zu foltern. In einem - zum Teil bewusst geschürten - Klima der Angst, in dem sich nicht einmal mehr die Mächtigsten unverletzlich fühlen, können die Menschen leicht davon überzeugt werden, dass der Preis für die Sicherheit die Aufgabe von persönlichen Freiheiten ist. Aber es gibt keine schlüssigen Nachweise, dass eine derartige Politik zu mehr Sicherheit führt. Im Gegenteil zeigen zahlreiche Beispiele, wie die Überbewertung von Sicherheitsinteressen die Welt nicht sicherer sondern unsicherer gemacht hat. Der Schutz der Menschenrechte darf nicht zugunsten einer trügerischen inneren Sicherheit relativiert werden. Wirkliche, nachhaltige Sicherheit kann nur erreicht werden durch die volle Achtung der Menschenrechte aller Menschen.

\section{Menschenrechte als Grundlage menschlicher Sicherheit}

Sicherheitspolitik muss, wie im UN-Konzept »menschlicher Sicherheit « vorgesehen, immer im Zusammenhang mit Menschenrechtsschutz gesehen werden. Das ist keine Selbstverständlichkeit. Das Ziel von amnesty international ist eine Welt, in der allen Menschen die in der Allgemeinen Erklärung der Menschenrechte und anderen internationalen Menschenrechtsinstrumenten kodifizierten Rechte gewährt werden. Der Grundgedanke des Konzepts menschlicher Sicherheit ist bereits in der UN-Charta mit dem Bekenntnis zu einem »besseren Lebensstandard in größerer Freiheit« angelegt. Die in

\footnotetext{
* Barbara Lochbihler ist Generalsekretärin der deutschen Sektion von amnesty international. Der Beitrag ist eine aktualisierte Fassung eines Vortrags am Institut für Friedensforschung und Sicherheitspolitik an der Universität Hamburg (IFSH) am 12. Oktober 2005.
}

der Allgemeinen Erklärung der Menschenrechte weiterentwickelte »Freiheit von Furcht und Not« entspricht den Kernaussagen der beiden grundlegenden Menschenrechtspakte von 1966. Die weltweite Geltung aller Menschenrechte ist in verschiedensten Konventionen und Erklärungen immer wieder bekräftigt worden. Die Abschlusserklärung der Wiener Weltmenschenrechtskonferenz von 1993 stellt den »universellen Charakter « der Menschenrechte außer Frage und akzeptiert damit keine Kulturrelativität. Die Erklärung bestätigt, »alle Menschenrechte sind allgemeingültig, unteilbar, bedingen einander und hängen miteinander zusammen«. Das heißt, politische, bürgerliche, wirtschaftliche, soziale und kulturelle Rechte bilden eine Einheit und müssen gemeinsam implementiert werden. In der Allgemeinen Erklärung der Menschenrechte sind auch wirtschaftliche, soziale und kulturelle Menschenrechte festgeschrieben. Sie sind weniger bekannt als die politischen und bürgerlichen Rechte, aber nicht weniger verbindlich und nicht weniger wichtig. Dazu zählen u. a. das Recht auf Bildung, auf Gesundheit und auf einen »angemessenen Lebensstandard «, was das Recht auf ausreichende Nahrung, Bekleidung, Wohnraum und soziale Leistungen einschließt. Es ist eine der Herausforderungen der Menschenrechtsarbeit, die Umsetzung dieser Rechte voranzutreiben und auch dafür zu sorgen, dass sie einklagbar werden.

Die Wiener Deklaration von 1993 bekräftigt auch die Verpflichtung der Staaten, den universellen Respekt für die Menschenrechte zu fördern und die Einhaltung der Menschenrechte zu sichern und durchzusetzen. Diese Verpflichtung gilt für alle Mitgliedstaaten der UN, unabhängig von der kulturellen Perspektive. Jeder Einzelne ist zum Genuss der Menschenrechte berechtigt, ohne Diskriminierung irgendeiner Art. Das Verbot der Diskriminierung ist eines der fundamentalen Prinzipien der Menschenrechte und schützt alle Menschen vor der Verleugnung ihrer Rechte. Universelle Menschenrechte zwingen somit keinen kulturellen Standard auf, sondern legen nur ein Mindestmaß des Schutzes der menschlichen Würde fest.

Als rechtlicher Standard, der von der UNO angenommen wurde, repräsentieren die universellen und unteilbaren Menschenrechte einen hart erkämpften Konsens der internationalen Gemeinschaft. Abgesehen von diesen minimalen Standards, die in diesen Rechten gewährt wurden, haben Staaten Möglichkeiten, die Menschenrechte entsprechend ihrer Kultur auszuformen. Doch obwohl der Anspruch auf universelle Gültigkeit der Menschenrechte überzeugend aus dem Völkerrecht 
abgeleitet werden kann, behalten sich einige Regierungen immer noch vor, sie nur insoweit umzusetzen, wie sie der von ihnen interpretierten Kultur oder Religion entsprechen.

\section{Menschenrechtsberichterstattung als Beitrag zur Prävention gewalttätiger Konflikte}

Menschenrechtsarbeit und Krisen- und Konfliktarbeit sind eng miteinander verbunden. Im Bereich der Prävention liegt der Schwerpunkt auf einer objektiven Menschenrechtsberichterstattung zu anhaltenden Spannungen und potenziellen Konfliktherden. Es mangelt selten an derartigen Berichten, doch sie müssen ernst genommen und ausgewertet werden. Es gibt zuwenig ernsthafte Versuche von Seiten der Politik, von einer »early warning « zu einer »early action « zu kommen. Dabei kann eine gute, das heißt auf gut recherchierten Fakten basierende Berichterstattung zur Menschenrechtssituation sehr nützlich sein. Mit der Veröffentlichung von Fakten zu Massakern an Zivilisten beispielsweise wird allen beteiligten Parteien ein Signal gegeben, dass ihre Taten nicht verborgen bleiben und sie für derartige Gräuel zur Verantwortung gezogen werden. Weiter kann durch genaue und schnelle Menschenrechtsuntersuchungen Kriegspropaganda und Gerüchten gegengesteuert werden, die einen Konflikt gegebenenfalls noch mehr aufheizen können. Durch Menschenrechtsberichte können Verantwortlichkeiten und Zusammenhänge für Menschenrechtsverletzungen aufgezeigt werden. Dies soll eine Warnung an alle Akteure sein, die Regeln einzuhalten.

Der Jahresbericht von amnesty international erscheint inzwischen in mehreren Sprachen rund um den Globus. Er ist ein umfassendes Nachschlagewerk zur Menschenrechtslage weltweit. Wer ihn liest, erhält gut dokumentierte Informationen zu den wesentlichen Entwicklungen im jeweils vergangenen Jahr. Hinzu kommen zahlreiche Länderberichte, mit denen wir uns immer wieder an die Weltöffentlichkeit wenden. Wir fordern von den Regierungen ein, sich auf ihre ureigenste Aufgabe zu besinnen: Sie haben die Menschenrechte ihrer Bürger und Bürgerinnen zu schützen, zu fördern und aktiv dazu beizutragen, dass Menschenrechtsverbrechen verhindert oder - wenn sie geschehen - aufgeklärt und die Verantwortlichen zur Rechenschaft gezogen werden.

\section{Menschenrechtsarbeit unter neuen »sicher- heitspolitischen Rahmenbedingungen«}

Die so genannten »sicherheitspolitischen Rahmenbedingungen«, unter denen wir heute Menschenrechtsarbeit leisten, haben sich seit dem Ende des »Kalten Krieges « in vielerlei Hinsicht geändert. Die Kriege und gewaltsamen Konflikte von heute bedeuten auch für die Menschenrechtsarbeit andere Herausforderungen. Die Konsequenzen daraus sind jedoch letztlich die gleichen wie schon bei der Verabschiedung der Allgemeinen Erklärung der Menschenrechte: Sicherheit für alle setzt Menschenrechte für alle voraus.

Die Konflikt- oder Kriegssituationen, in denen Sicherheitserwägungen - scheinbar - dem umfassenden Menschenrechts- schutz entgegenstehen, lassen sich grob in drei Kategorien einteilen:

- die Situation so genannter »failing states«, bei denen es internen wie externen Akteuren vorrangig um die (Wieder)Erlangung von Sicherheit im Sinne von Stabilität geht;

- innerstaatliche gewaltsame Konflikte zwischen staatlichen Akteuren, Rebellengruppen und zumeist auch paramilitärischen Akteuren, bei denen die Menschenrechte oft der Wiederherstellung des staatlichen Gewaltmonopols, gleichgesetzt mit der Gewährleistung von Sicherheit, untergeordnet werden;

- und der weltweite so genannte »Krieg gegen den Terrorismus «, in dem auch in Rechtsstaaten zugunsten vermeintlicher Sicherheit zunehmend Menschenrechtsstandards ausgehöhlt werden.

\subsection{Einige menschenrechtspolitische Grundlagen für nachhaltige Sicherheit}

Im Hinblick auf die ersten beiden Komplexe sollen zunächst drei - keineswegs neue - menschenrechtliche Aspekte hervorgehoben werden, weil sie sowohl für die Beendigung als auch die Prävention schwerer Menschenrechtsverletzungen eine besondere Rolle spielen und deshalb auch von amnesty international immer wieder thematisiert werden: die Straflosigkeit schwerer Menschenrechtsverbrechen, die Rüstungsexporte in Krisenländer und die Rolle von Frauen in bewaffneten Konflikten.

Nach einem akuten Konflikt ebenso wie bei jahrelangen gewaltsamen Auseinandersetzungen innerhalb eines Staates verflüchtigt sich die internationale Aufmerksamkeit schnell, doch gerade dann ist weitere konsequente Menschenrechtspolitik gefordert. Etwa durch den Aufbau rechtsstaatlicher Justizsysteme und die Einrichtung von nationalen Institutionen, die Menschenrechte fördern und schützen. Verschiedene Formen, wie mit Tätern von Menschenrechtsverletzungen im Prozess der Versöhnung umgegangen werden soll, sind denkbar. Gesellschaftliche Friedens- und Versöhnungsprozesse und die Aufarbeitung von Menschenrechtsverbrechen stehen oft in einem Spannungsverhältnis zueinander. Doch wenn nachhaltiges friedliches und damit sicheres Zusammenleben wieder möglich sein soll, darf die Frage nach Gerechtigkeit nicht beiseite gewischt werden. Alle unsere Erfahrungen zeigen, dass die ernsthafte und sorgfältige Aufarbeitung von Menschenrechtsverletzungen - sowohl von individuellen wie systematischen Übergriffen - ein ganz zentraler Aspekt gesellschaftlicher Versöhnungsprozesse wie auch der Verhinderung zukünftiger Menschenrechtsverletzungen ist. Aus Sicht der Opfer steht dabei die materielle Entschädigung oft gar nicht an erster Stelle der Wiedergutmachung, hier spielen auch die formelle Anerkennung ihrer Leiden und eine offizielle Entschuldigung eine nicht zu unterschätzende Rolle. Wir sind hier erst am Anfang und müssen intensiv Untersuchungen vorantreiben, welche Formen in den jeweiligen Gesellschaften nachhaltig gewirkt haben. An verschiedensten Beispielen wird jedoch immer wieder deutlich, dass die Benennung der Täter und deren strafrechtliche Verantwortung vor Gericht eine wesentliche Voraussetzung 
für die nachhaltige Gewährleistung von Menschenrechten und Sicherheit sind. In El Salvador etwa herrschen trotz offiziellen Endes des zwölfjährigen Bürgerkriegs nicht Frieden und Sicherheit, sondern Gewalt und Instabilität durch anhaltende Straflosigkeit. Das Amnestiegesetz von 1993 verhindert bis heute eine gerichtliche Aufarbeitung der massiven Menschenrechtsverletzungen während des Bürgerkrieges und die Entschädigung der Opfer. In Kolumbien ist ein Ende des schon vierzigjährigen Bürgerkrieges auch deshalb nicht in Sicht, weil die derzeitige Kampagne zur Demobilisierung der Paramilitärs einhergeht mit einer weitgehenden Straffreiheit von Verantwortlichen für Verschwindenlassen, Folter, Vertreibungen und extralegale Hinrichtungen und das Gesetz für »Gerechtigkeit und Frieden « zudem die Rechte der Opfer auf Wahrheit, Gerechtigkeit und Entschädigung missachtet.

Ein großer Schritt vorwärts bei der Beendigung schwerer Menschenrechtsverletzungen, Kriegsverbrechen und Verbrechen gegen die Menschlichkeit ist dagegen der Internationale Strafgerichtshof. amnesty international setzt sich deshalb für die weltweite Ratifizierung des Römischen Statuts und die Umsetzung des Prinzips universeller Gerichtsbarkeit in den nationalen Rechtsordnungen ein. Und wir widersprechen immer wieder den Versuchen, Soldaten und Personal von UN-Friedensmissionen aus Nichtvertragsstaaten von der Gerichtsbarkeit des Intentionalen Strafgerichtshofes auszunehmen. Gerade Repräsentanten der Vereinten Nationen dürfen keine Immunität genießen, wenn sie sich schwerer oder systematischer Menschenrechtsverletzungen schuldig gemacht haben. Auch die jahrelang aussichtslos erscheinende Überstellung hoher Politiker und Militärs bis hin zu Staatspräsidenten an internationale oder nationale Strafgerichte, wie nach Pinochet und Milosevic zuletzt im Falle Charles Taylor, signalisiert den Tätern immer deutlicher, dass sie nicht mehr damit rechnen können, dass ihre Verbrechen ungesühnt bleiben.

Die Verhinderung des Exports von militärischen Gütern und Sicherheitsausrüstungen sowie von Kleinwaffen und leichten Waffen, wenn davon ausgegangen werden kann, dass sie zur Konflikteskalation und Menschenrechtsverletzungen beitragen, ist eine weitere menschenrechtliche Grundvoraussetzung für die Gewährleistung von individueller wie kollektiver Sicherheit. Weltweit werden jedes Jahr eine halbe Million Menschen durch Waffengewalt getötet - ein Mensch pro Minute. Regierungen, die lautstark vor der Bedrohung durch Massenvernichtungswaffen warnen, tragen gleichzeitig durch den hemmungslosen Transfer von konventionellen Waffen, darunter Klein- und Leichtwaffen, zur Eskalation von Konflikten rund um den Erdball bei. amnesty international, Oxfam International und das »Internationale Aktionsnetzwerk zu Kleinwaffen « führen deshalb unter dem Motto »Waffen unter Kontrolle« in über 60 Ländern eine Kampagne gegen unkontrollierten Waffenhandel durch. Vorrangiges Ziel ist die strikte Kontrolle und Transparenz aller Rüstungstransfers durch ein rechtlich verbindliches internationales Abkommen. Ein solches Abkommen soll einheitliche Standards für den Waffenhandel schaffen und alle Exporte verbieten, die zur Verletzung der Menschenrechte und des humanitären Völkerrechts beitragen - eine globale Lösung für ein globales Problem.
Grundvoraussetzung für die Gewährleistung nachhaltiger Sicherheit ist schließlich auch die Beendigung von Gewalt gegen Frauen und Mädchen in bewaffneten Konflikten gleichermaßen wie im familiären Kontext sowie die stärkere Einbindung von Frauen in Friedensverhandlungen und -prozesse. Die Empfehlungen der vom Sicherheitsrat schon im Jahr 2000 beschlossenen Resolution 1325 sehen vor, dass Frauen auf allen nationalen, regionalen und internationalen Entscheidungsebenen der Prävention, Konfliktbeilegung und Friedenskonsolidierung verstärkt eingebunden werden und eine Gender-Perspektive in allen Prozessen der Friedenssicherung systematisch integriert und implementiert wird.

\subsection{Die Relativierung elementarer Menschen- rechtsstandards im »Kampf gegen den Terro- rismus«}

Nach wie vor missbrauchen die Staaten den »Krieg gegen den Terror « als Rechtfertigung, um Menschen »verschwinden « zu lassen, um sie ohne ordentliches Verfahren zu inhaftieren, um sie zu misshandeln und zu foltern. Vor allem nach den Anschlägen vom 11. September 2001 in den USA sowie den Anschlägen vom 11. März 2004 in Madrid und den beiden Anschlägen in London im Juli 2005 sind auf internationaler, europäischer und nationaler Ebene zahlreiche Maßnahmen ergriffen worden, um den »Terrorismus « zu bekämpfen. Viele dieser Maßnahmen greifen in grundlegende Menschenrechte ein und führen zu einer Erosion des Systems des Menschenrechtsschutzes, wie er sich nach dem Ende des Zweiten Weltkrieges entwickelt hat. Staaten wie die USA und Großbritannien stellen das absolute Folterverbot zur Disposition. Großbritannien hat »Terrorismusverdächtige« ohne Anklageerhebung unrechtmäßig in Haft gehalten und plant offen einen Verstoß gegen das absolute Folterverbot, indem es »Terrorverdächtige« auch in Länder abschieben oder ausliefern will, in denen ihnen Folter droht. Durch Einholen diplomatischer Zusicherungen der Zielstaaten, dass die »Terrorismusverdächtigen « nicht gefoltert würden, wollen sich immer mehr Regierungen absichern und auch in Staaten abschieben und ausliefern, in denen gefoltert wird.

Nach den Anschlägen vom 11. September 2001 sind in den USA fundamentale internationale Menschenrechtsstandards zunehmend ausgehebelt worden: Die US-Regierung begann schrittweise, das Verbot von Folter und erniedrigender Behandlung im Namen der Sicherheit aufzuweichen. Anfang 2002 gab das Verteidigungsministerium eine Richtlinie heraus, die "Individuen der al-Qaida und der Taliban« das Anrecht auf den Status als Kriegsgefangene aberkennt. Im Spätsommer 2002 wurde der Begriff Folter neu definiert und Verhörmethoden erlaubt, die nach international vereinbarten Kriterien als Folter gelten. Es wurde in Abrede gestellt, dass der Schutzbereich des Folterverbots sich auch auf Fälle erstreckt, in denen eine grausame, unmenschliche und erniedrigende Behandlung und Bestrafung erfolgt. Im Frühjahr 2003 genehmigte Verteidigungsminister Rumsfeld die Anwendung von 24 »widerstandsbrechenden « Techniken und behielt es sich vor, gegebenenfalls »zusätzliche Verhörtechniken« im Einzelfall 
zu genehmigen. Weltweite Kritik am Lager Guantánamo verhallte lange ungehört. Die entsetzlichen Folterbilder aus Abu Ghraib, Irak, brachten die US-Regierung dann 2004 erstmals unter ernsthaften Druck. Berichte über »Geisterhäftlinge« und geheime Haftzentren folgten. Terrorverdächtige wurden durch die CIA verschleppt und an Staaten überstellt, die von den USA sonst wegen Folter und anderen Menschenrechtsverletzungen kritisiert werden. Schließlich verkündete die US-Außenministerin im Dezember 2005, die UN-Antifolterkonvention "gilt für US-Personal, wo immer es sich aufhält, in den USA oder im Ausland «. Und wenig später gab Präsident Bush seinen Widerstand gegen das entsprechende Gesetz auf, das Folter und Misshandlung auch im Ausland verbietet und auch den Geheimdiensten untersagt. Dabei hat er sich jedoch augenscheinlich eine juristische Hintertür offen gehalten, wie ein öffentlich gewordenes »Statement des Präsidenten « verdeutlicht. Aus diesem wird ersichtlich, dass sich die Regierung mit Blick auf das Antifoltergesetz einen nicht überprüfbaren Interpretationsspielraum zubilligt.

amnesty international hat wiederholt öffentlich die USA für die von ihr zu verantwortenden Menschenrechtsverletzungen scharf kritisiert. Zwei Jahre nach den skandalösen Folterfällen im Abu Ghraib Gefängnis in Bagdad warten wir weiter auf eine unabhängige und umfassende Untersuchung. Bisher untersucht und richtet sich der Verdächtige selbst. Kein Wunder, dass Verantwortliche auf höherer Ebene entweder nicht oder nur geringfügig belangt werden. Nach wie vor haben internationale Experten keinen Zugang zu allen von den USA kontrollierten Haftzentren, von Baghram in Afghanistan bis Guantánamo. Wir wissen, dass dort überall gefoltert wurde und vermutlich noch wird.

Gleichzeitig müssen wir erleben, wie auch bei uns in Deutschland über die Zulässigkeit von Folter diskutiert wird. Ein zivilisatorischer Konsens, der als unumstößlich galt, wird in Frage gestellt. »Nein zur Folter. Ja zum Rechtsstaat « ist das Motto, unter dem amnesty international der Debatte zur Relativierung des Folterverbots begegnet. Es ist auch der Titel eines Appells, mit dem sich Persönlichkeiten aus allen Bereichen der Gesellschaft zusammen mit amnesty international im Mai 2005 gegen die Aufweichung des Folterverbots ausgesprochen haben. In diesem Aufruf heißt es: »Weite Teile der Bevölkerung wie auch hochrangige Politiker und Juristen wollen Folter in begründeten Einzelfällen zulassen. Neben Kindesentführung gibt hier vor allem der ,Krieg gegen den Terror' das Stichwort. Mehrere Neukommentierungen zum Grundgesetz sowie zur Strafprozessordnung haben in jüngster Zeit der Aufweichung des Folterverbotes das Wort geredet. Die Menschenwürde soll abwägbar, ihre Unverfügbarkeit aufgehoben werden. «Es kann jedoch für den Staat keine Rechtfertigung geben, Menschen in seinem Gewahrsam zu foltern oder zu misshandeln. Nach deutschem wie nach Völkerrecht ist Folter, auch in Zeiten von Notstand, Krieg oder besonderer Gefahr, absolut verboten. Folter ist durch keinen Zweck, keine Absicht, ein anderes Rechtsgut schützen zu wollen - und sei es das Recht auf Leben - zu rechtfertigen. Folter verletzt die Menschenwürde und diese ist unantastbar. Denn der Schutz der Menschenwürde ist die Grundlage eines Rechtsstaats. Demnach können die Bürgerinnen und Bürger darauf vertrauen und im Zweifelsfall rechtlich geltend machen, dass der Staat seine Gewalt innerhalb dieser Grenzen ausübt. Dieser sicher geglaubte Konsens scheint derzeit jedoch verloren zu gehen. Viele Regierungen, auch in Europa, weichen vor allem unter dem Vorwand des »Kriegs gegen den Terror« das Prinzip des absoluten Folterverbots auf. Die bisher geltenden Definitionen von Foltermaßnahmen werden in Frage gestellt, an ihrer Stelle werden neue, verwischende Umschreibungen gesucht. Regierungen bemühen sich zudem nicht oder nur unzureichend, die für Folter Verantwortlichen zur Rechenschaft zu ziehen. Vielmehr scheint Folter - ohne dass sie so benannt würde - im Sicherheitskonzept des Anti-Terror-Kampfes salonfähig zu werden. Gerade in Deutschland resultiert das absolute Folterverbot auch aus einer historischen Erfahrung, die noch kein Menschenalter zurückliegt. Dass diese Erfahrung eine Lehre für die Gegenwart sein muss, scheint in unserer Gesellschaft nicht mehr selbstverständlich zu sein. Wie bereits in unserem Antifolteraufruf festgehalten, gilt jedoch: Wer Folter - und sei es in Ausnahmefällen - zulassen will, der ruft nach einem anderen als dem freiheitlich-demokratischen Rechtsstaat.

In den vergangenen Monaten haben weitere menschenrechtlich äußerst fragwürdige Sachverhalte die Diskussion in Deutschland bestimmt. Als im November 2005 die Verschleppung des Deutschen Khaled El Masri durch die CIA in den Talkshows diskutiert wurde, war die Meinung einhellig. Politiker aller Parteien verdammten die so genannten »renditions«, also die Verschleppung von Terrorverdächtigen. Solche Entführungen seien unter allen Umständen illegal. Die geäußerte Empörung auf dem Höhepunkt der öffentlichen Aufmerksamkeit steht allerdings in seltsamem Gegensatz zu den nur mangelhaften Anstrengungen, das illegale Vorgehen umfassend aufzuklären und womöglich zu verhindern. Vertreter der Bundesregierung selbst betreiben zudem im Namen der Terrorismusbekämpfung eine Politik, die eine schrittweise Aufweichung von Menschenrechtsstandards bewirkt. So musste Bundesinnenminister Wolfgang Schäuble am 14. Dezember 2005 vor dem Bundestag zugeben, dass Beamte des Bundesnachrichtendienstes einen Gefangenen in Guantánamo und Beamte des Bundeskriminalamtes einen Gefangenen in Syrien befragt haben. Es wurde bald deutlich, dass es sich um Murat Kurnaz, einen türkischen Staatsangehörigen, der in Deutschland lebt, und den Deutsch-Syrer Mohammed Haider Zammar handelt. Bemerkenswert ist das Eingeständnis des Bundesinnenministers schon deshalb, weil die Bundesregierung es im Fall Kurnaz lange Zeit abgelehnt hatte, sich für die Rechte des völkerrechtswidrig Inhaftierten einzusetzen, weil er kein deutscher Staatsangehöriger sei. Im Zusammenhang mit der Inhaftierung Zammars in Syrien hatte das Auswärtige Amt nach Auskunft seiner Anwältin auf Nachfrage mitgeteilt, dass es keinen Kontakt zu dem Gefangenen herstellen konnte. Umso mehr befremdet es nunmehr zu erfahren, dass beide Personen offenbar zur Gewinnung von Informationen im Kampf gegen den Terrorismus von deutschen Behörden »abgeschöpft « worden sind.

Innenminister Schäuble hat auch betont, dass im Rahmen der Terrorismusbekämpfung auch Erkenntnisse genutzt werden sollten, bei denen die Gefahr besteht, dass sie unter Folter erlangt worden sind. Verwendbar sollen Informationen derart 
zweifelhaften Ursprungs zwar nicht im Strafverfahren sein, wohl aber als Grundlage weiterer Ermittlungen oder im Rahmen der Gefahrenabwehr. Im Ergebnis würden die deutschen Sicherheitsbehörden damit zu »Profiteuren der Folter«. Der Folter wird damit bewusst oder unbewusst Vorschub geleistet und das auch von der Regierung so versicherte absolute Folterverbot aufgeweicht.

Die Menschenrechte gelten ausnahmslos auch im Kampf gegen den Terror, versichert die Bundesregierung stets öffentlich. Zur Umsetzung dieser Versicherung zählt es, Menschenrechte von Partnerländern einzufordern, die offensichtlich dagegen verstoßen; dazu zählt, Menschenrechtsverletzungen vorbehaltslos aufzuklären, insbesondere dort, wo eine deutsche Beteiligung gegeben zu sein scheint; und dazu zählt auch, den eigenen Behörden, die wesentlich am Kampf gegen den Terror beteiligt sind, exakte Vorgaben zu machen, wo ein Verstoß gegen Menschenrechte beginnt. Bei all diesen Themen gibt es für die Bundesregierung noch viel zu tun.

Es gibt für mich keinen Zweifel: Die Urheber terroristischer Anschläge müssen gefasst und vor Gericht gestellt werden. Die entsetzlichen Gewaltakte bewaffneter politischer Gruppen sind Ausdruck der völligen Missachtung fundamentalster Grundsätze der Menschlichkeit. Und: Die Staaten haben das Recht und die Pflicht, ihre Bürgerinnen und Bürger - soweit möglich - vor weiteren Anschlägen zu schützen. Menschenrechtsverletzungen sind jedoch kein Mittel der Terrorbekämpfung.

Viele Versuche, größere Sicherheit vor nicht auszuschließenden Anschlägen zu erreichen, haben vor allem die Aushöhlung international und verbindlich geltender Menschenrechtsstandards zur Folge. Von der Veränderung der gesellschaftlichen Diskurse in Zeiten des Terrorismus ganz zu schweigen. Unverkennbar ist mit dem Kampf gegen den Terrorismus die Logik des »gerechten Krieges « in das politische und rechtliche Den- ken zurückgekehrt. Hier muss die Menschenrechtsbewegung nicht nur im Einzelfall protestieren, sondern muss verstärkt und langfristig die Diskussion zum Schutz der Menschenrechte gegenüber staatlichen Interessen führen. Auch ein so genannter »Terrorist « verliert seine Menschenrechte nicht. Es macht moderne Rechtsstaaten aus, dass sie diesen Grundsatz respektieren, so schwer es im Einzelnen auch fallen mag.

Eine Politik, die Sicherheit auf Kosten der Menschenrechte erreichen will, erreicht ihr Ziel nicht. Damit treibt sie Menschen in die Arme derer, die eine freie, auf Grundrechten für alle beruhende Gesellschaft ohnehin ablehnen. Gerade in »schwierigen Zeiten« ist der Einsatz für die Menschenrechte unerlässlich.

\section{Schlussbemerkung}

Die Grundprinzipien der Allgemeinen Erklärung der Menschenrechte sind heute wichtiger denn je. Wirkliche individuelle und kollektive Sicherheit entsteht erst, wenn Menschenrechte respektiert und geachtet werden. Dieser elementare Grundsatz gilt in gleicher Weise für die nationale wie die internationale Ebene. Menschenrechtsstandards stellen das absolut notwendige Minimum dar, um die Sicherheit und Integrität von Individuen vor Machtmissbrauch zu schützen. Sie sind keine Gefälligkeitserklärungen der Regierungen. Ohne den Schutz des Rechtsstaates, inklusive Mechanismen zur Rechenschaftspflicht, können Aktionen gegen Verdächtige zu schweren Menschenrechtsverletzungen führen. Die Herausforderung für die Staaten besteht darin, die Sicherheit der Bürger nicht auf Kosten der Menschenrechte zu verbessern, sondern vielmehr sicherzustellen, dass alle Menschen in den Genuss des gesamten Spektrums ihrer elementaren Rechte gelangen.

\title{
Lateinamerika
}

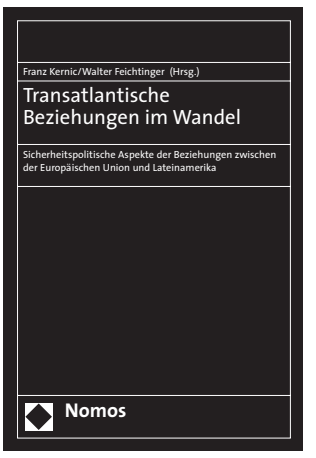

\author{
Transatlantische Beziehungen im Wandel \\ Sicherheitspolitische Aspeke der Beziehungen zwischen der Europäischen Union und Lateinamerika \\ Herausgegeben von Franz Kernic und Walter Feichtinger \\ 2006, 261 S., brosch., 49,- €, ISBN 3-8329-2015-3
}

Autoren aus Europa, den USA und Lateinamerika beziehen Stellung und analysieren verschiedene Dimensionen der Sicherheitskooperation bzw. strategischen Partnerschaft. Der Bogen spannt sich von einer Analyse der strukturellen Wandlungen im internationalen System sowie geopolitischen Überlegungen bis hin zur Untersuchung zentraler Aspekte der Sicherheitspolitik und Security Sector Reform in ausgewählten lateinamerikanischen Staaten.

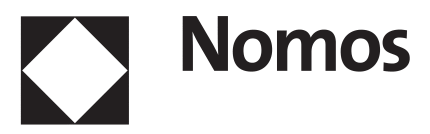

Bitte bestellen Sie bei Ihrer Buchhandlung oder bei: Nomos Verlagsgesellschaft 76520 Baden-Baden | www.nomos.de 